\title{
Atención de los síndromes coronarios agudos durante la contingencia sanitaria por brote de SARS-CoV-2
}

\author{
Care of acute coronary syndromes during the health contingency due to a SARS-CoV-2 \\ outbreak
}

\begin{abstract}
Yigal Piña-Reyna ${ }^{1,2 *}$, Andrés García-Rincón ${ }^{2,3}$, Patricio H. Ortiz-Fernández P ${ }^{1,2}$, Marco A. Alcocer-Gamba ${ }^{2,4,5}$, Pedro Gutiérrez-Fajardo6,7, José A. Merino-Rajme ${ }^{2,5,8}$ y Gustavo Reyes-Terán ${ }^{9}$

${ }^{1}$ Instituto Nacional de Cardiología "Ignacio Chávez", Secretaria de Salud, Ciudad de México, México; 2Sociedad de Cardiología Intervencionista de México; ${ }^{3}$ Hospital de Especialidades Dr. Antonio Fraga Mouret del Centro Médico Nacional La Raza, Instituto Mexicano del Seguro Social, Ciudad de México, México, Sociedad de Cardiología Intervencionista de México; ${ }^{4}$ Instituto de Corazón de Querétaro, Facultad de Medicina, UAQ, Querétaro; ${ }^{5}$ Sociedad Mexicana de Cardiología; ${ }^{6}$ Hospitales Mac Bernardette y Sanatorio San Francisco de Asis, Guadalajara, Jalisco, México; ${ }^{7}$ Asociación Nacional de Cardiólogos de México; ${ }^{8}$ Servicio de Hemodinamia, Centro Médico Nacional 20 de Noviembre, Instituto de Seguridad y Servicios Sociales de los Trabajadores del Estado, Ciudad de México, México. Asociación Nacional de Cardiólogos al Servicio de los Trabajadores del Estado (ANCISSSTE); ${ }^{9}$ Centro de Investigación en Enfermedades Infecciosas, Instituto Nacional de Enfermedades Respiratorias Ismael Cosió Villegas, Secretaría de Salud. Ciudad de México, México, Comisión Coordinadora de Institutos Nacionales de Salud y Hospitales de Alta Especialidad (CCINSHAE)
\end{abstract}

\section{Resumen}

Las comunicaciones acumuladas en las últimas semanas dejan claro que no existe un acuerdo para definir la mejor estrategia de tratamiento en los pacientes con un síndrome coronario agudo (SICA). En los pacientes que se presentan con un infarto agudo del miocardio con elevación del segmento ST (IAMCESST) se ha sugerido privilegiar la fibrinólisis (FL) sobre la intervención coronaria percutánea primaria (ICPp), reservando el ICP para los casos de FL fallidar',2; sin embargo algunas sociedades han mantenido la indicación de la ICPp como el método de repercusión de elecciónr ${ }^{3}$. En los SICA sin elevación del segmento ST (SICASESST) las recomendaciones son muy similares, favoreciendo el tratamiento medico sobre el intervencionismo coronario percutáneo, en este subgrupo de pacientes'. Varias sociedades consideran el estado de contagio, en particular en IOS SICASESST, para decidir que estrategia de repercusión seguir ${ }^{3}$. Anticipando que la curva epidemiológica en México será similar a la observada en la mayoría de los países, recomendamos continuar la atención de los pacientes con SICA, las salas de cateterismo deben mantener su funcionamiento.

Palabras clave: Síndrome coronario agudo. Contigencia sanitaria. Brote COVID-19.

\begin{abstract}
The communications accumulated in the last weeks make it clear that there is no agreement to define the best treatment strategy in patients with acute coronary syndrome (SICA). In patients presenting with an acute myocardial infarction with ST-segment elevation (IAMCESST), it has been suggested to favor fibrinolysis (FL) over primary percutaneous coronary in-
\end{abstract}

Correspondencia:

*Yigal Piña Reyna

E-mail: yigalpr@yahoo.com
Disponible en internet: 26-05-2020 Arch Cardiol Mex. 2020;90(Supl):33-35

www.archivoscardiologia.com 1405-9940/๑ 2020 Instituto Nacional de Cardiología Ignacio Chávez. Publicado por Permanyer. Este es un artículo open access bajo la licencia CC BY-NC-ND (http://creativecommons.org/licenses/by-nc-nd/4.0/). 
tervention (PCI), reserving ICP for cases of failed $F L^{1,2}$; however, some societies have maintained the indication of the ICPp as the repercussion method of choice ${ }^{3}$. In SICAs without ST segment elevation (SICASESST) the recommendations are very similar, favoring medical treatment over percutaneous coronary intervention in this subgroup of patients ${ }^{1}$. Several companies consider the contagion status, particularly in the SICASESST, to decide which repercussion follow ${ }^{3}$. Anticipating that the epidemiological curve in Mexico will be similar to that observed in most countries, we recommend continuing the care of patients with SICA, the catheterization rooms must maintain their operation.

Key words: Acute coronary syndrome. Health contingency. COVID-19 outbreak.

Las comunicaciones acumuladas en las últimas semanas dejan claro que no existe un acuerdo para definir la mejor estrategia de tratamiento en los pacientes con un síndrome coronario agudo (SICA). En los pacientes que se presentan con un infarto agudo del miocardio con elevación del segmento ST (IAMCESST) se ha sugerido privilegiar la fibrinolisis (FL) sobre la intervención coronaria percutánea primaria (ICPp), reservando el ICP para los casos de FL fallidar ${ }^{1,2}$; sin embargo algunas sociedades han mantenido la indicación de la ICPp como el método de repercusión de elección ${ }^{3}$. En los SICA sin elevación del segmento ST (SICASESST) las recomendaciones son muy similares, favoreciendo el tratamiento medico sobre el intervencionismo coronario percutáneo, en este subgrupo de pacientes ${ }^{1}$. Varias sociedades consideran el estado de contagio, en particular en los SICASESST, para decidir que estrategia de repercusión seguir ${ }^{3}$.

Anticipando que la curva epidemiológica en México será similar a la observada en la mayoría de los países, recomendamos continuar la atención de los pacientes con SICA, las salas de cateterismo deben mantener su funcionamiento.

\section{IAMCESST}

En los pacientes habituales con un IAMCESST, o los que son sospechosos/confirmados con COVID-19 y que se complican con un IAMCESST, se debe mantener la estrategia farmaco invasiva habitual en México, aceptando que son necesarias algunas modificaciones. Los centros médicos con salas de cateterismo deben favorecer la ICPp sobre la fibrinolisis, justificado en la mayor tasa de éxito, menor riesgo de complicaciones y en una menor estancia hospitalaria. La FL es un opción de reperfusión adecuada en los centros sin sala de cateterismo y en los pacientes con neumonía o afectación grave por COVID-19. A diferencia de la estrategia fármaco invasiva convencional se debe evitar el traslado de pacientes estables con fibrinolisis exitosa para cateterismo electivo temprano, estos pacientes se seguirán y estudiarán en un tiempo posterior. Únicamente se debe trasladar a los pacientes con fibrinolisis fallida, en especial los pacientes inestables. La FL también se puede considerar una alternativa en centros con salas de hemodinamia cuando el hospital enfrenta una demanda elevada y creciente de casos sospechosos o confirmados.

\section{SICASESST}

En los pacientes que se presentan o desarrollan un SICASESST. y son de bajo riesgo, independientemente de la presencia o ausencia de SARS-CoV-2, pueden ser dados de alta del hospital e ir a cateterismo en un segundo tiempo. Si se considera necesario documentar la anatomía coronaria antes del alta, una opción es realizar angiotomografía coronaria y en función de los hallazgos plantear la ICP o dar el alta a domicilio. Los pacientes de riesgo moderado o alto o los que se inestabilizan durante el curso del tratamiento médico conservador, se deben llevar a sala de cateterismo independientemente de su estado de contagio. En los pacientes con enfermedad de múltiples vasos, es preferible acortar su estancia y privilegiar la revascularización por ICP sobre la cirugía de revascularización coronaria.

Finalmente, las autoridades de cada hospital decidirán el método de reperfusión más adecuado de acuerdo con el equipo, personal y recursos existentes. Será necesario ajustar periódicamente la estrategia de reperfusión en función del comportamiento de la curva epidemiológica.

\section{Responsabilidades éticas}

Protección de personas y animales. Los autores declaran que para esta investigación no se han realizado experimentos en seres humanos ni en animales.

Confidencialidad de los datos. Los autores declaran que han seguido los protocolos de su centro de trabajo sobre la publicación de datos de pacientes. 
Derecho a la privacidad y consentimiento informado. Los autores declaran que en este artículo no aparecen datos de pacientes.

\section{Bibliografía}

1. Zeng J, Huang J, Pan L. How to balance acute myocardial infarction and COVID-19: the protocols from Sichuan Provincial People's Hospital. Intensive Care Med. 2020.
2. Welt FGP, Shah PB, Aronow HD, et al. Catheterization laboratory considerations during the coronavirus (COVID-19) pandemic: From ACC's Interventional Council and SCAI. J Am Coll Cardiol. 2020. https://doi. org/10.1016/j.jacc.2020.03.021

3. Romaguera R, Cruz-González I, Jurado-Román A, et al. Consideraciones sobre el abordaje invasivo de la cardiopatía isquémica y estructural durante el brote de coronavirus COVID-19. Documento de consenso de la Asociación de Cardiología Intervencionista y la Asociación de Cardiopatía Isquémica y Cuidados Agudos Cardiovasculares de la Sociedad Española de Cardiología. REC Interv Cardiol. 2020. https://doi. org/10.24875/RECIC.M20000119 